University of Nebraska - Lincoln

DigitalCommons@University of Nebraska - Lincoln

March 2001

\title{
The Endocrine-Genetic Basis of Life-History Variation: The Relationship Between The Ecdysteroid Titer and Morph-Specific Reproduction in the Wing-Polymorphic Cricket Gryllus Firmus
}

\author{
Anthony J. Zera \\ University of Nebraska - Lincoln, azera1@unl.edu \\ Justin Bottsford \\ University of Nebraska - Lincoln
}

Follow this and additional works at: https://digitalcommons.unl.edu/bioscizera

Part of the Microbiology Commons

Zera, Anthony J. and Bottsford, Justin, "The Endocrine-Genetic Basis of Life-History Variation: The Relationship Between The Ecdysteroid Titer and Morph-Specific Reproduction in the Wing-Polymorphic Cricket Gryllus Firmus" (2001). Anthony Zera Publications. 14.

https://digitalcommons.unl.edu/bioscizera/14

This Article is brought to you for free and open access by the Papers in the Biological Sciences at DigitalCommons@University of Nebraska - Lincoln. It has been accepted for inclusion in Anthony Zera Publications by an authorized administrator of DigitalCommons@University of Nebraska - Lincoln. 
Published in Evolution 55:3 (March 2001), pp. 538-549. Copyright (C) 2001 The Society for the Study of Evolution.

Published by Blackwell Publishing Inc. Used by permission. DOI: 10.1554/0014-3820(2001)055[0538:TEGBOL]2.0.CO;2

Submitted June 21, 2000; accepted October 6, 2000.

\title{
The Endocrine-Genetic Basis of Life-History Variation: The Relationship Between The Ecdysteroid Titer and Morph-Specific Reproduction in the Wing-Polymorphic Cricket Gryllus Firmus
}

\author{
Anthony J. Zera* and Justin Bottsford \\ School of Biological Sciences, University of Nebraska-Lincoln, Lincoln, Nebraska 68588 \\ *Corresponding author. E-mail: azera@unlserve.unl.edu
}

\begin{abstract}
The hormonal basis of variation in life-history traits is a poorly studied topic in life-history evolution. An important step in identifying the endocrine-genetic causes of life-history variation is documenting statistical and functional associations between hormone titers and genotypes/phenotypes that vary in life-history traits. To this end, we compared the blood ecdysteroid titer and the mass of the ovaries during the first week of adulthood among a flight-capable morph and two flightless morphs of the wing-polymorphic cricket Gryllus firmus. Ecdysteroids are a group of structurally related hormones that regulate many important aspects of reproduction in insects. Both the ecdysteroid titer and ovarian mass were significantly higher in each of two flightless morphs compared with the flight-capable morph throughout the first week of adulthood. Genetically based differences in the ecdysteroid titer and ovarian mass between morphs from different selected lines were similar to phenotypically based differences among morphs from the same control (unselected) lines. By day 7 of adulthood, ovaries were typically 200$400 \%$ larger and the ecdysteroid titer was $60-300 \%$ higher in flightless versus the flight-capable morph. In addition, highly significant, positive, phenotypic correlations were observed between the ecdysteroid titer and ovarian mass in pooled samples of the two flightless and flight-capable crickets from control lines or from selected lines. The ecdysteroid titer was sufficiently elevated in the flightless morphs to account for their elevated ovarian growth. This is the first direct documentation that naturally occurring phenotypes/genotypes that differ in early fecundity, a key life-history trait, also differ phenotypically and genetically in the titer of a key reproductive hormone that potentially regulates that trait.
\end{abstract}

Keywords: Crickets, ecdysteroids, genetic variation, hormones, life history, trade-off, wing polymorphism

The physiological causes of life-history variation and trade-offs has been an important topic in evolutionary biology for more than six decades (Fisher 1930; Townsend and Calow 1981; Stearns 1992; Zera et al. 1998; Zera and Harshman 2001). Until recently, most research on this topic has focused on energetic aspects, such as the differential allocation of nutrients to reproduction versus somatic maintenance in phenotypes, genotypes, or species that vary in life histories (Calow 1979; Townsend and Calow 1981; Djawdan et al. 1996; Zera and Harshman 2001). The importance of variation in physiological regulation as a functional cause of variation in individual life-history traits and trade-offs between traits has received much less attention.

A priori, there are several reasons to suspect that hormonal variation might be an important physiological cause of life-history variation or covariation (Ketterson and Nolan 1992, 1999; Sinervo and Svensson 1998; Zera et al. 1998; Zera and Harshman 2001). First, life-history traits, which determine age-specific patterns of growth, sexual maturity, and longevity, are precisely those organismal features that are known to be tightly controlled by hormones (Kerkut and Gilbert 1985; Martin 1985; Norris 1996). Thus, it is reasonable to suspect that variation in hormonal regulation might underlie observed variation in individual life-history traits. Second, because hormones typically affect many traits simultaneously (Ketterson and Nolan 1992, 1999), they are prime candidates as physiological causes of correlations (e.g., trade-offs, covariation) between life-history traits. The importance of obtaining detailed endocrine information in functional studies of life-history evolution has been repeatedly stressed (e.g., Stearns 1989; Ketterson and Nolan 1992, 1999). Yet, few studies have directly documented phenotypic variation in the concentration (titer) of hormones that potentially underlie life-history variation. Moreover, evolutionary-genetic studies of the hormonal underpinnings of life-history variation are just beginning to be undertaken (see Discussion).

Wing polymorphism is common in many insect groups and involves dramatic variation in a wide variety of life-history traits (Harrison 1980; Roff 1986; Zera and Denno 1997). One morph has fully developed wings and flight muscles and is capable of flight. Alternate morphs, either with reduced wings and reduced flight muscles or with reduced flight muscles alone, cannot fly. In both types of flightless morph, ovarian growth is substantially greater (often $200-400 \%$ ) compared with the flight-capable morph during the first week of adulthood (Zera and Denno 1997; Zera et al. 1997; see Results). That is, flight capability trades off with early reproduction.

We have been using wing polymorphism in species of Gryllus (crickets) to study evolutionary modification of the endocrine mechanisms that regulate life-history traits (Zera and Tiebel 1989; Zera et al. 1989; Zera and Denno 1997; Zera and Huang 1999; Zera and Cisper 2001; Zera and Harshman 2001). Thus far, virtually all of our endocrine studies have focused on juvenile hormone $(\mathrm{JH})$, a known regulator of many key aspects of reproduction in insects (e.g., synthesis and uptake of yolk protein; Kerkut and Gilbert 1985; Wyatt and Davey 1996). We have documented that adult morphs of G. firmus that differ in ovarian growth also differ in the hemolymph (blood) titer of this hormone. Furthermore, the $\mathrm{JH}$ titer is phenotypically correlated with ovarian mass in this species (Cisper et al. 2000; Zera and Cisper 2001). 
These data clearly suggest that differences in early reproduction between wing morphs of G. firmus result, at least in part, from underlying differences in the $\mathrm{JH}$ titer. However, ovarian growth is influenced by many hormones other than $\mathrm{JH}$, and the importance of differences in the titers of these hormones as functional causes of phenotypic and genetic differences between morphs in early reproduction is unknown.

Ecdysteroids are a group of structurally related steroid hormones that regulate many aspects of adult reproduction and development in insects (Nijhout 1994; Tawfik et al. 1997; Strambi et al. 1997; references therein). Variation in the ecdysteroid titer has been implicated as a physiological cause of alternate morph development in the wing-polymorphic cricket, G. rubens (Zera et al. 1989; Zera and Denno 1997) and morph-specific reproduction in the phase-polyphenic locust Schistocerca gregaria (Tawfik et al. 1997). In the present study, we compared the hemolymph ecdysteroid titer among a flight-capable and two flightless morphs of the cricket, G. firmus, during the first week in adulthood, when morphs differ dramatically in ovarian growth. Endocrine measurements were undertaken in control lines and in lines that had been artificially selected for alternate morphs. The overall purpose of this study was to determine whether phenotypic and genetic differences in a key life-history trait (early reproduction) were associated with and could be the functional consequence of underlying phenotypic and genetic differences in the ecdysteroid titer.

\section{Materials and Methods}

\section{Stocks, Morphs, Rearing Conditions, and Artificial Selection Study}

Gryllus firmus, the sand cricket, exists in the southeastern United States as a long-winged (LW) morph, some of which are capable of flight, or as a short-winged (SW) morph that is obligatory flightless (Veazy et al. 1976; Zera et al. 1997). Except in a few rare cases, all SW females molt into adults with white, nonfunctional, underdeveloped flight muscles. All LW females initially have fully developed, functional (pink) flight muscles at or shortly after the adult molt. Individuals with functional flight muscles are denoted as LW(f). Upon onset of ovarian growth, some LW(f) individuals histolyze their flight muscles, thus becoming flightless (denoted LW(h); see below and Zera et al. 1997).

The G. firmus females used in the present study were derived from stocks selected for the LW or SW morph or from unselected control lines. Full details of the selection study will be reported elsewhere. Briefly, three blocks, each of which contained a LWselected (L) line, a SW-selected (S) line, and an unselected control (C) line, were derived from the same generation of a single base population (Fig. 1). The base colony used for the selection study was founded from 30 gravid females collected in Gainesville, Florida during the summer of 1995. The number following the line designation denotes the block to which it belongs (e.g., L-1, LW selected stock of block 1). L lines were propagated by breeding only LW males and LW females, whereas the S lines were propagated by breeding only SW males and SW females each generation. LW and SW males and females were chosen at random to propagate the $\mathrm{C}$ lines. In the present study, crickets were used during generations 4-6 of the selection study. During these generations wing morph (LW vs. SW) differs genetical-

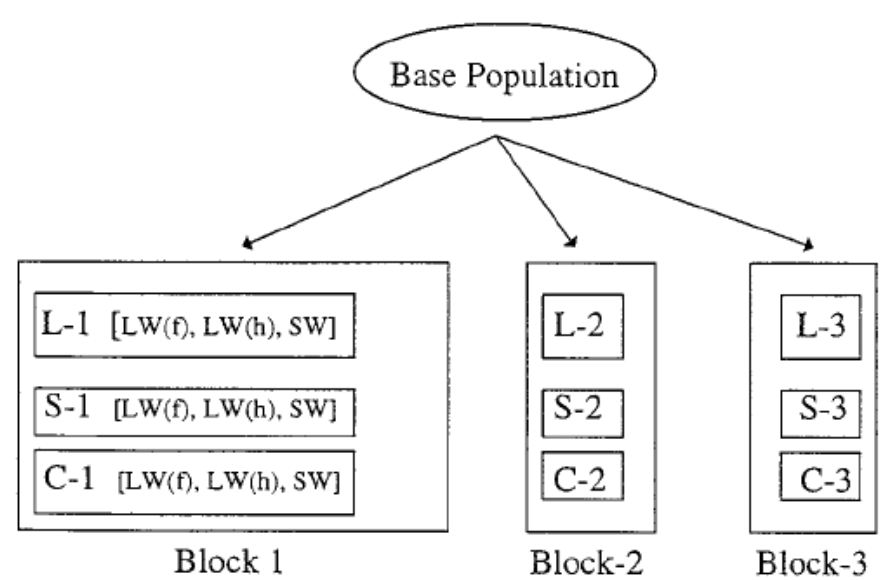

Figure 1. Design of the artificial selection study on wing morph in Gryllus firmus. L, S, and C denote, respectively, lines selected for the longwinged (LW), short-winged (SW) morph, or unselected controls. Block refers to a replicate selection trial. LW(f) denotes a long-winged morph with large, functional flight muscles; LW(h) denotes a long-winged morph with small, histolyzed flight muscles.

ly between the L and S lines (Zera and Huang 1999). Grand mean frequency of the LW morph (average of the three line means \pm $\mathrm{SE}$ for a particular generation) ranged from $0.81 \pm 0.04$ to 0.71 \pm 0.01 for generations $4-6$ in the L lines and from $0.29 \pm 0.04$ to $0.34 \pm 0.02$ in the $\mathrm{S}$ lines. Grand mean frequencies of the $\mathrm{LW}$ morph ranged from $0.66 \pm 0.06$ to $0.47 \pm 0.08$ during generations 4-6 in the two control lines.

Crickets were reared at $28^{\circ} \mathrm{C}$ under a 16:8 L:D photoperiod. All individuals of all lines were fed the same diets and were reared under the same densities, as described for $G$. assimilis (Zera et al. 1998), with the following exceptions. Crickets were switched from the dry to the wet diet during the penultimate stadium and were raised at a density of 80 and 40 crickets per 10gallon aquarium during the penultimate and last stadium, respectively. Crickets used for experiments were checked for molting at 24-h intervals and equal numbers of newly molted males and females were held together at a density of four to six per 1-gallon box or eight to 12 per 3 -gallon box. Because no oviposition material was provided, females retained all of their eggs.

\section{Morphological and Reproductive Characteristics of Dispersing and Nondispersing Morphs}

Wing length (long or short) and flight muscle phenotype (reduced or fully developed), which together define the three morphsLW(f), LW(h) and SW (see above)-were scored in each G. firmus whose ecdysteroid titer was measured. The length of the wings is clearly discontinuous and can easily be classified as long or short. Flight muscle color is strongly correlated with muscle size (fully developed muscles are pink; reduced muscles [underdeveloped in SW, histolyzed in LW] are white) and was used to classify LW individuals as either LW(f) or LW(h) (see Zera et al. 1997). On days 3-7, all SW females had reduced muscles as was observed in an earlier study (Zera et al. 1997, unpublished data). Both ovaries were dissected from each female, whose ecdysteroid titer was measured, and the ovaries were weighed. 


\section{Quantification of Hemolymph Ecdysteroid Titers}

Hemolymph (blood) ecdysteroid titers were measured in female morphs that were 3,5 , or 7 days old (molt to adult $=$ day 0 ). These days were chosen because they span the period of development during which ovaries first begin to grow to a time when ovarian mass is substantially greater in either flightless morph, SW or LW(h), compared with the flight-capable morph, LW(f) (see Cisper et al. 2000; Results). Between $12.5 \mu \mathrm{l}$ and $25.0 \mu \mathrm{l}$ of hemolymph were collected from individual females and blown into $300 \mu \mathrm{l}$ of $90 \%$ methanol in water. Samples were sonicated for 3-5 sec and extracted twice with $600 \mu \mathrm{l}$ hexane (see Cisper et al. 2000). Methanol extracts were stored at $-80^{\circ} \mathrm{C}$ until they were subjected to radioimmunoassay (RIA). Ecdysteroids were quantified using the polyclonal ecdysteroid antiserum from the "A" series of Horn et al. (1976). This antiserum was produced in the laboratory of W. E. Bollenbacher (University of North Carolina, Chapel Hill), and was kindly distributed by E. S. Chang (Bodega Marine Laboratory, Bodega Bay, California). The RIA protocol used in the present study was a modified protocol of Warren et al. (1984) and Chang and O’Connor (1979). Briefly, an aliquot of the hemolymph methanol extract was placed in a test tube and the solvent was evaporated in vacuo. To each tube was added $200 \mu \mathrm{l}$ of approximately $25,000-30,000$ DPM of tritiated ecdysone (New England Nuclear, Boston, MA), and 200 $\mu \mathrm{l}$ of anti-ecdysone antibody, each in borate buffer $(0.1 \mathrm{M}$ boric acid, 0.1 $\mathrm{M}$ sodium tetraborate, and $0.075 \mathrm{M} \mathrm{Na} \mathrm{Cl}, \mathrm{pH} 8.4$ ). In the various control assays, either antibody or hemolymph extract was not added. Finally, $20 \mu \mathrm{l}$ of protein A solution (Sigma Chemical Co., St. Louis, MO, P-7155) was added to each tube to allow precipitation of antibody-ligand complex after incubation. Tubes were vortexed for $5 \mathrm{sec}$, covered with plastic wrap, and incubated for $18 \mathrm{~h}$ at $4^{\circ} \mathrm{C}$. After this time, tubes were centrifuged at $3000 \times \mathrm{g}$ for $15 \mathrm{~min}$, and the supernatant was drawn off and discarded. The pellet was resuspended in $100 \mu \mathrm{l}$ of water and the suspension was added to $2 \mathrm{ml}$ of Ready Safe scintillation cocktail (Beckman Instruments, Fullerton, CA). DPMs were quantified by liquid scintillation spectrometry. On each RIA run, a series of ecdysone standards, ranging from $8000.0 \mathrm{pg}$ to $15.6 \mathrm{pg}$, were added instead of the hemolymph extracts, so that a standard curve could be calculated. Ecdysteroid concentrations were estimated by nonlinear regression (Prism 2.0, GraphPad, Inc, San Diego, CA).

\section{Phenotypic Differences in the Ecdysteroid Titer among Wing Morphs: Statistical Analyses and Hypotheses Tested}

Phenotypic differences among morphs with respect to the ecdysteroid titer were tested by factorial analysis of variance (ANOVA; Sokal and Rohlf 1989). Main effects were morph [LW(f), LW(h), and SW], block, and age (days 3, 5, and 7 of adulthood). Because we were mainly interested in determining whether the ecdysteroid titer differed between specific morphs, the overall ANOVA was followed by comparisons of each pair of morphs. Comparison between LW(f) and SW morphs tests whether morphs that differ in the development of both wings and flight muscles, during the juvenile stage, differ in their adult ecdysteroid titer. Comparison between LW(f) and LW(h) tests whether morphs that differ in flight muscle phenotype, generated during adulthood by histolysis, differ in the ecdysteroid titer. Finally, comparisons between LW(h) and SW tests whether flightless morphs produced by different developmental pathways (blockage of muscle growth vs. histolysis) that occur during different life-cycle stages (adults vs. juveniles) differ in the ecdysteroid titer. These comparisons were planned, a priori contrasts; thus, probabilities were not adjusted for experimentwide error. Prior to ANOVA, ecdysteroid titers were log-transformed to normalize their distributions and equalize the variances among groups.

Three types of phenotypic differences among morphs with respect to the ecdysteroid titer were analyzed. The first involved comparisons among the three morphs taken from control lines. This analysis estimates phenotypic differences in the ecdysteroid titer among morphs due to genetic and environmental factors in the base population. Because of the expense of materials and the amount of labor required, it was not feasible to measure ecdysteroid titers in morphs from all three control lines. Thus, ecdysteroid titers were not measured in one of the three controls $(\mathrm{C}$ 2 ; eliminated at random). ANOVAs of ecdysteroid titer differences among morphs were preformed separately for each of the two control lines. This was done because of a marginally significant morph $\times$ block interaction (see Results).

Genetic analyses of the ecdysteroid titer (see below) involved comparisons between mean hormone titers between morphs taken from different selected lines. These ecdysteroid titer data were also analyzed for phenotypic variation among individual morphs. This second type of phenotypic analysis was the same as the analysis of ecdysteroid titer variation among the three morphs derived from the same control lines, except that LW(f) and LW(h) individuals were taken from L lines, whereas SW individuals were derived from $\mathrm{S}$ lines. In the three-way ANOVA of these data, no significant two- or three-way interactions were observed among main effects (morph, age, or block; $P>0.4$ in all cases). Consequently, a pooled error mean square was computed as follows (see Sokal and Rohlf 1989). The combined sums of squares for all interaction and error terms was divided by the pooled degrees of freedom for these terms. $F$-ratios for the main effects were calculated by dividing the mean square for that effect by the pooled mean square for the error and interaction terms. Measurement of ecdysteroid titers in the same morph derived from selected or control lines also allowed us to test for the effect of genetic background on ecdysteroid titer. This third analysis of phenotypic variation was done by ANOVA in which line type (selected or control line) was included as a categorical factor in addition to morph, age, and block.

\section{Analyses of Phenotypic Variation in Ovarian Mass and Correla- tions between Ovarian Mass and Ecdysteroid Titers}

Phenotypic differences in ovarian mass among the three morphs derived from the same control lines or among the LW(f) and LW(h) morphs from the L lines and the SW morph from the S lines were ascertained by Kruskal-Wallis (K-W) tests of ovarian mass scaled to body mass (percent body wet mass). Ovarian mass was not analyzed by ANCOVA, with body mass as a covariate, 
because of various statistical problems such as nonnormal distributions of ovarian mass and the existence of various treatment $\times$ covariate interactions. Because the analysis of ratios is often problematical (Packard and Bordman 1987), we performed a parallel set of K-W tests using unscaled ovarian mass. Distributions of ovarian mass and ovarian mass scaled to body mass were essentially identical, as were results of K-W tests performed on either scaled or unscaled data (see Results). Associations between ovarian mass scaled to body mass and log ecdysteroid titer were determined by Spearman correlations. These correlations were very similar to those estimated with unscaled ovarian masses (see Results). Probabilities for these correlations reported in the Results are corrected for multiple comparisons estimated from the same dataset.

\section{Analyses of Genetic Differences in Wing Morph, Ovarian Mass, and Ecdysteroid Titer}

A major goal of the present study was to determine if morphs that differ genetically in flight capability and ovarian growth also differ genetically in the ecdysteroid titer. Genetically based differences in flight capability should be manifested as a consistently elevated frequency of the LW(f) phenotype in the three $\mathrm{L}$ lines compared with the three $\mathrm{S}$ lines. Similarly, mean ovarian mass and mean ecdysteroid titer should be consistently higher in SW individuals from the $\mathrm{S}$ lines relative to $\mathrm{LW}(\mathrm{f})$ individuals from the L lines. Each of these hypotheses was tested by a paired $t$-test similar to analyses described in Zera and Huang (1999) and Rose et al. (1996). With respect to the ecdysteroid titer, the difference between the mean value for the ecdysteroid titer measured in flight-capable [LW(f)] individuals from an L line versus the mean value measured in flightless (SW) individuals from the $\mathrm{S}$ line of the same block was computed. This was repeated for each of the three blocks. The mean and standard error of these differences was then used to compute a $t$-statistic with two $d f[=2$ (number of blocks -1 )]. Pseudoreplication (i.e., inflation of degrees of freedom) results when more than one datapoint per selected line is used in analysis of genetic differences between lines (see Rose et al. 1996). Consequently, in the present study, paired $t$-tests were performed on least-squared means of titers averaged over the three days of adulthood or separate $t$ tests were performed on ecdysteroid titer data for each day of adulthood.

Similar $t$-tests were performed on the arcsine-transformed frequency of the LW(f) phenotype in the L and S lines, and standardized ovarian masses (as arcsine percent body mass) in LW(f) females from the L lines and SW females from the S lines. We did not score LW(f) and LW(h) morphs in the S lines; thus, the frequency of the LW(f) morph in an L line was actually compared to the frequency of the LW morph in the corresponding $\mathrm{S}$ line. The frequency of the LW morph must be at least equal to and is almost certainly greater than the frequency of the LW(f) morph (see Results). Therefore, this constitutes a conservative test for differences in the frequency of the LW(f) morph between L and S lines. A previous study documented that the SW morph differs genetically between (i.e., its frequency is consistently higher in) S versus L lines (Zera and Huang 1999).
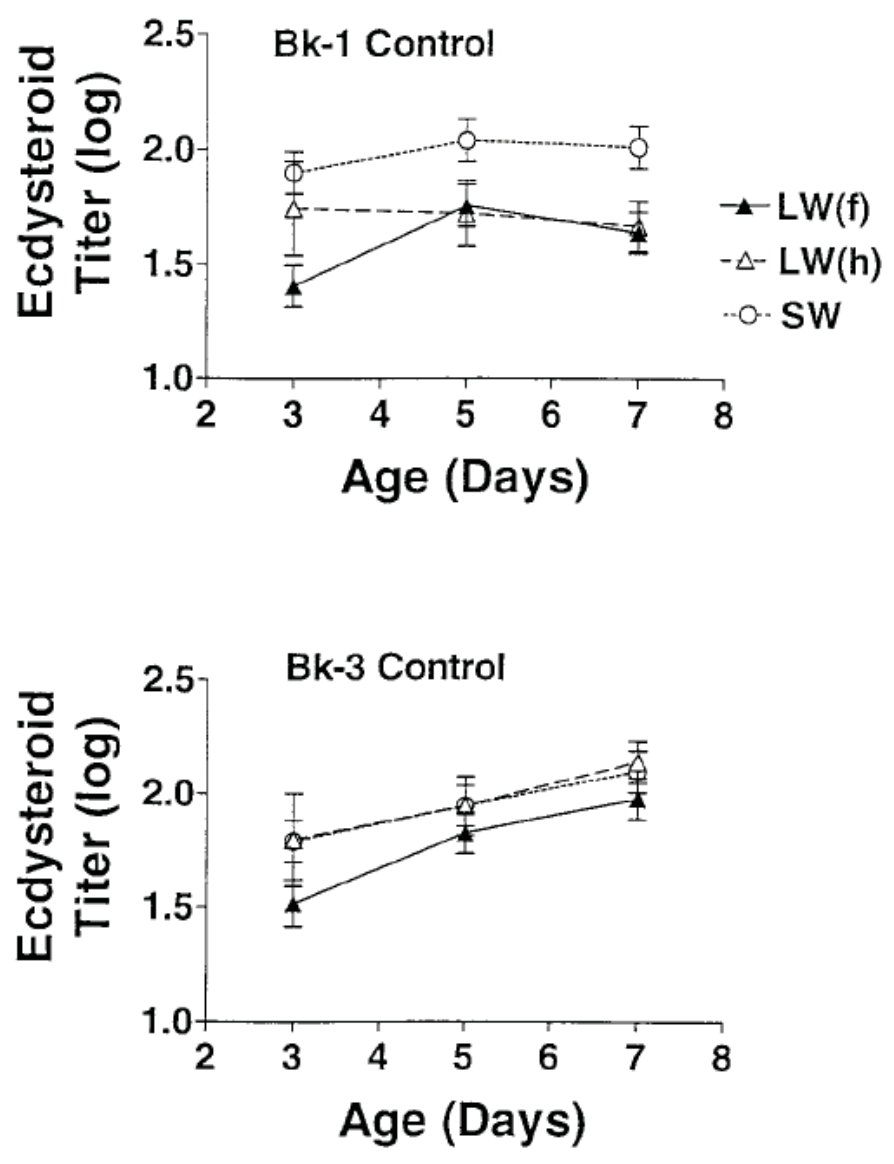

Figure 2. Phenotypic differences in the ecdysteroid titer among wing and flight-muscle morphs of Gryllus firmus during adulthood in control lines. Symbols are means and bars are SEM. LW(f), LW(h), and SW refer, respectively, to long-winged individuals with functional flight muscles, long-winged individuals with histolyzed (reduced) flight muscles, and short-winged females with underdeveloped (reduced) flight muscles. Age refers to day after molt to adult (day 0). Means were based on seven to 10 females [LW(f) or SW], four to 10 females [day 5 or day $7 \mathrm{LW}(\mathrm{h})$ ], or two females [day $3 \mathrm{LW}(\mathrm{h})$ ].

\section{Results}

\section{Phenotypic Variation in the Ecdysteroid Titer among Morphs}

Mean ( \pm SEM) hemolymph ecdysteroid titers for the three morphs of the C-1 and C-3 (control) lines of G. firmus, measured on days 3, 5, and 7 of adulthood, are presented in Figure 2. Ecdysteroid titers were not measured in the other (C-2) control line (see Methods). Because of a marginally significant interaction between block and morph $\left(F_{2,127}=2.44, P=0.09\right)$, data for control lines from each block were analyzed separately. Significant differences were observed in the ecdysteroid titer among the three morphs in each line (block 1: $F_{2,61}=9.7, P<0.001$; block $3: F_{2,66}$ $=5.6, P<0.01)$. Titers also differed with age in block $3\left(F_{2,66}=\right.$ $13.9, P<0.001)$, but not in block $1\left(F_{2,61}=1.47, P>0.2\right)$ No significant interactions were observed between age and morph in either of these tests or in any subsequent paired comparison $(P>$ 0.3 in each test). When pairs of morphs were compared, the ecdysteroid titer was significantly lower in the flight-capable LW(f) 

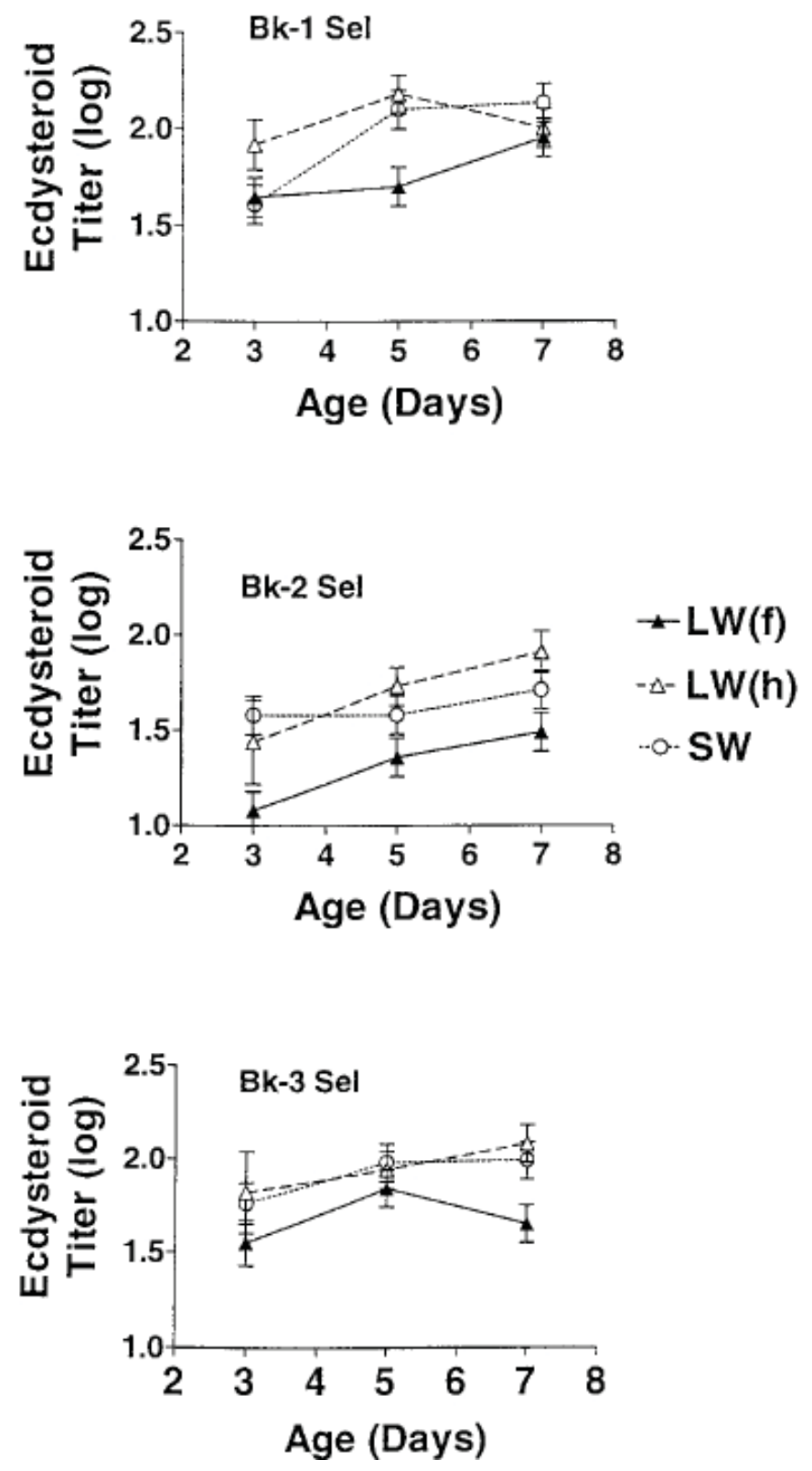

Figure 3. Phenotypic differences in the ecdysteroid titer among wing and flight-muscle morphs of Gryllus firmus during adulthood in selected lines. Symbols are the same as in Figure 2. LW(f) and LW(h) females came from the LW-selected lines, whereas SW females came from the $\mathrm{SW}$-selected lines. Means were based on eight to 10 females [LW(f) or $\mathrm{SW}$ ], five to 10 females [day 5 or day $7 \mathrm{LW}(\mathrm{h})$ ], or two to six females [day $3 \mathrm{LW}(\mathrm{h})$ ].

versus the flightless SW morph in each block (block 1: $F_{1,53}=$ 23.5, $P<0.001$; block 3: $\left.F_{1,52}=9.7, P<0.005\right)$. The ecdysteroid titer was also significantly lower in the LW(f), compared to the other flightless morph $[\mathrm{LW}(\mathrm{h})]$ in block $3\left(F_{1,39}=6.4, P<\right.$ $0.02)$, but not in block $1\left(F_{1,34}=0.83, P>0.3\right.$; see Fig. 2$)$. Finally, the ecdysteroid titer either did not differ significantly between the two flightless morphs (block $3: F_{1,41}=0.04, P>0.8$ ), or only differed marginally (block $1: F_{1,35}=4.4, P=0.04$ ).

Mean ( \pm SEM) ecdysteroid titers for the LW(f) and LW(h) morphs derived from the L lines and the SW morph derived from the $\mathrm{S}$ lines are presented in Figure 3. As was the case for crickets derived from the control lines, ecdysteroid titers differed among morphs $\left(F_{2,227}=19.55, P<0.001\right)$ and days of adulthood $\left(F_{2,227}\right.$ $=12.44, P<0.001)$. Significant differences were also observed among the three blocks $\left(F_{2,227}=27.65, P<0.001\right)$. No significant interactions were observed between any main effect (ANOVA, $P$ $>0.4$ in each test). In pairwise comparisons between morphs over all ages and blocks, the ecdysteroid titer was significantly lower in the flight-capable LW(f) versus the flightless SW morph $\left(F_{1,154}\right.$ $=23.2, P<0.001)$, or flightless LW(h) morph $\left(F_{1,128}=27.6, P<\right.$ $0.001)$. However, the ecdysteroid titer did not differ significantly between the two flightless morphs $\left(F_{1,132}=1.50, P=0.22\right)$. This is evident in Figure 3, where the ecdysteroid titers for the two flightless morphs are very similar on most days.

\section{Effect of Genetic Background on Morph-Specific Ecdysteroid Titers}

Only a small influence in genetic background was observed when ecdysteroid titers were compared between the same morph taken from control versus selected lines. Because of an interaction between block and line type (i.e., control vs. selected line), data were analyzed separately for titers in blocks 1 and 3 (hormone titers were not measured in C-2 lines). For block 3 lines, there was no overall effect of line type on the ecdysteroid titer $\left(F_{1,139}\right.$ $=0.62, P>0.3)$, nor were any significant interactions observed that involved morph or line type ( $P>0.2$ in all cases). In addition, separate comparisons of a particular morph between control and selected lines were all nonsignificant $(P>0.3$ in each of three ANOVAs). In the overall ANOVA and in the three ANOVAs of individual morphs, significant differences in the ecdysteroid titer were observed among the three days of adulthood $(P<0.05$ in each ANOVA). Because the ANOVA of block 1 lines yielded a significant interaction between morph and line type $\left(F_{2,141}=\right.$ $4.48, P<0.025)$, we analyzed differences in the JH titer between control and selected lines separately for each morph. No significant difference was found for the SW morph from the control versus selected lines $\left(F_{1,54}=0.51, P>0.4\right)$ and only slight differences were found for the LW(f) morph $\left(F_{1,54}=4.27, P=0.04\right.$; LS means [log-transformed titers] $=1.77 \pm 0.06$ [selected, $n=30$ ] and $1.60 \pm 0.06$ [control, $n=30]$ ). In contrast, the ecdysteroid titer was significantly elevated in selected versus control LW(h) $G$. firmus $\left(F_{1,32}=6.76, P<0.025 ;\right.$ LS means $=2.03 \pm 0.06$ [selected, $n=26]$ and $1.77 \pm 0.09$ [control, $n=12])$.

\section{Phenotypic Differences in Ovarian Mass among Morphs}

Ovaries grew considerably faster in either flightless morph [SW or LW(h)] compared with the flight-capable [LW(f)] morph during the first week of adulthood (Figs. 4, 5). This was the case whether comparisons were made among morphs derived from the same control lines (Fig. 4) or from the same or different selected lines (Fig. 5). On day 7 of adulthood, mean standardized ovarian mass (as \% body wet mass) ranged from $200-400 \%$ higher in either flightless morph compared to the flight capable LW(f) morph. In control lines (data pooled across lines), standardized ovarian mass was smaller in the flight-capable LW(f) compared with the flightless SW morph on each of days 3, 5, and 7 of adulthood $(P<0.005$ for each K-W test). Similarly, the LW(f) morph 

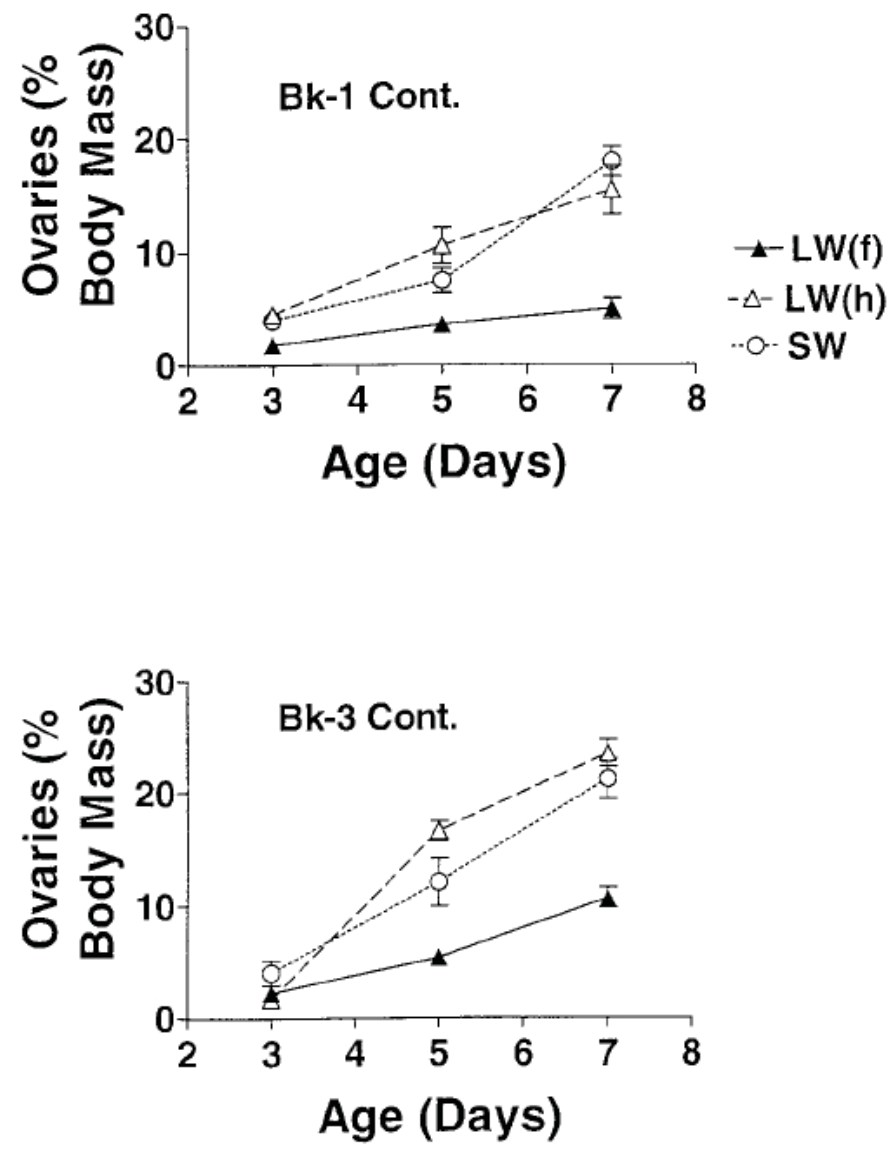

Figure 4. Phenotypic differences in ovarian mass among wing and flightmuscle morphs of Gryllus firmus in control lines. Ovarian wet mass was scaled to total body wet mass. Symbols and sample sizes are the same as those in Figure 2. Essentially identical patterns were obtained for raw ovarian masses (see Methods).

had smaller ovaries than the LW(h) morph on days 5 and 7 of adulthood $(P<0.005$ for each $\mathrm{K}-\mathrm{W}$ test), but not on day $3(\mathrm{~K}-\mathrm{W}$ test: $\left.\chi^{2}=0.88, P>0.3\right)$. In contrast, standardized ovarian mass did not differ significantly between the two flightless morphs, LW(h) and SW, on either days 3 or 7 of adulthood $(P>0.5$ for each $\mathrm{K}-\mathrm{W}$ test $)$ and only differed marginally on day $5\left(\chi^{2}=3.92\right.$, $P=0.05$ ). Because ovarian mass was nearly perfectly correlated with standardized ovarian mass (Spearman correlation, $r=0.99$, $n=144$ ), essentially identical results were obtained in a parallel series of K-W tests in which ovarian mass was compared among morphs (results not shown).

Similar phenotypic differences in standardized ovarian mass were observed among morphs taken from lines selected for the LW or SW morph [LW(f) and LW(h) morphs from the L lines and the SW morph from the S lines; Fig. 5]. As was the case for control lines, ovarian mass was nearly perfectly correlated with ovarian mass scaled to body mass for crickets derived from selected lines $(r=0.98, n=227)$. Thus, results of $\mathrm{K}-\mathrm{W}$ tests were the same irrespective of which measure of ovarian mass was compared between the morphs. Ovaries were significantly smaller in the flight-capable LW(f) compared with the SW morph on each day of adulthood $(P<0.005$ in each $\mathrm{K}-\mathrm{W}$ test; data pooled across the three L or three S lines) and were significantly smaller than ovaries in the flightless LW(h) morph on days 5 and 7 ( $P<0.005$ in each K-W test), but not day $3\left(\chi^{2}\right.$ $=2.8, P=0.09$ ). In contrast, ovarian masses either did not differ significantly or only differed marginally between the two flightless morphs $(P>0.3$ for two of three $\mathrm{K}-\mathrm{W}$ tests, and $P=0.05$ for one $\mathrm{K}-\mathrm{W}$ test).

\section{Phenotypic Correlations between the Ecdysteroid Titer and Ovarian Mass}

Bivariate distributions of ovarian mass standardized or unstandardized to body mass versus log ecdysteroid titer for morphs from control or selected lines are presented in Figure 6. Because of the very high correlation between standardized and unstandardized ovarian masses (see above), the two types of distributions within a line type (control or selected lines) were virtually identical (Fig. 6). Similarly, pairs of correlations between the ecdysteroid titer and either measure of ovarian mass always differed by less than $9 \%$. A highly significant, positive phenotypic correlation was observed between standardized ovarian mass and hemolymph ecdysteroid titer when all morphs of control lines were tested $(r=0.60, P<0.005, n=144)$. Similar correlations were observed when only LW(f) and SW morphs were tested ( $r=0.64, P<0.001, n=115)$ or when only LW(f) and LW(h) morphs were tested $(r=0.65, P<0.005, n=87)$. Highly significant positive phenotypic correlations were also observed between standardized ovarian mass and ecdysteroid titer measured in LW(f) and LW(h) morphs from the L lines and the SW morph from the S lines. This was the case for the three morphs tested together $(r=0.47, P<0.005, n=227)$, for LW(f) and SW morphs tested alone ( $r=0.44, P<0.005, n=$ $140)$, or for $\mathrm{LW}(\mathrm{f})$ and $\mathrm{LW}(\mathrm{h})$ morphs tested alone $(r=0.52, P$ $<0.005, n=140)$.

\section{Genetic Analyses of Wing Morph Frequencies, Ovarian Mass, and Ecdysteroid Titer}

Frequencies of wing and flight-muscle morphs scored in the control and selected lines on days 3, 5, and 7 of adulthood during generation six of selection are given in Table 1 . In each of the three blocks on each of the three days of adulthood the frequency of the LW(f) morph in an L line was significantly higher than the frequency of the LW morph in the corresponding $\mathrm{S}$ line of the same block $\left(P<0.025\right.$ [usually $P<0.01$ ] in each of nine $\chi^{2}$ tests $)$. Furthermore, on each of days 3, 5, and 7 of adulthood, the grand mean frequency of the LW(f) morph in the L lines was significantly higher than the grand mean frequency of the LW morph in the $\mathrm{S}$ lines (results of paired $t$-tests: day $3, t_{2}=15.05, P<0.005$; day $5, t_{2}=7.51, P<0.025$; day $\left.7, t_{2}=11.17, P<0.01\right)$. Thus, the LW(f) morph from L lines and the SW morph from S lines differ in genetic factors that control the expression of wings and flight muscles.

SW individuals from the S lines exhibited genetically elevated ovarian mass compared with $\mathrm{LW}(\mathrm{f})$ individuals from the $\mathrm{L}$ lines. In each of the three blocks, standardized ovarian mass was higher in SW females from an S line compared with LW(f) females from the corresponding $L$ line on each of the three days of adulthood $(P<0.005$ in each of nine K-W tests). Furthermore, 

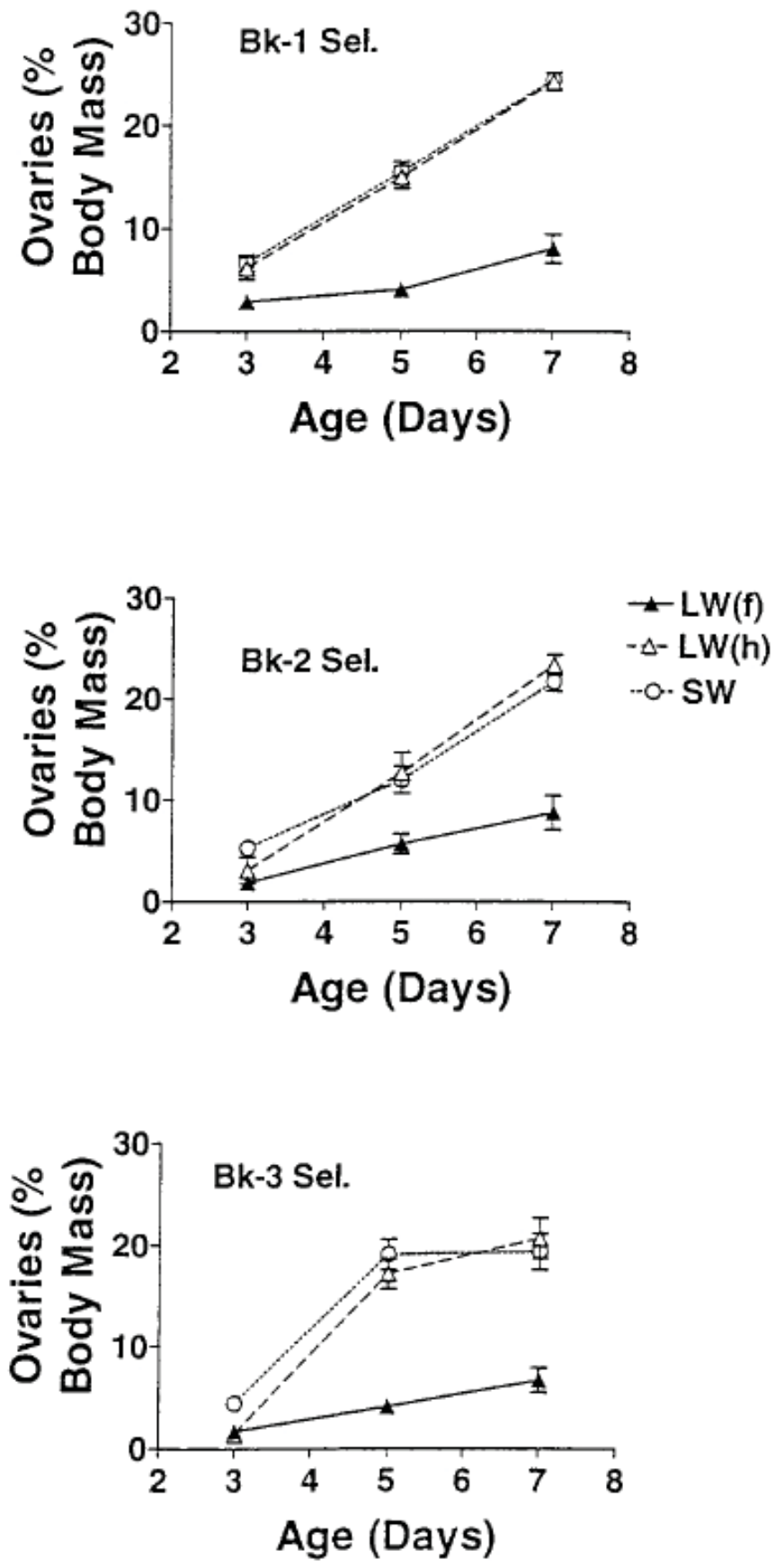

Figure 5. Phenotypic differences in ovarian mass among wing and flight muscle morphs of Gryllus firmus in selected lines. LW(f) and LW(h) females came from the LW-selected lines, whereas SW females came from the SW-selected lines. Symbols are the same as those in Figure 2, and sample sizes are the same as those in Figure 3. Ovarian mass was scaled to total body mass. Essentially identical patterns were obtained for raw ovarian masses.

the grand mean ovarian mass (arcsine-transformed means of Fig. 5 ) was significantly higher in these SW versus $\mathrm{LW}(\mathrm{f})$ females on day $3\left(t_{2}=21.1, P<0.005\right)$, day $5\left(t_{2}=4.76, P<0.05\right)$, day 7 $\left(t_{2}=14.4, P<0.005\right)$, and when averaged over the three days of adulthood $\left(t_{2}=11.2, P<0.01\right.$; Fig. 7$)$.

In each of the three blocks, the mean ecdysteroid titer (LS means averaged over the three days of adulthood) was signif- icantly higher in the flightless SW morph from an S line compared with the flight capable LW(f) morph from the corresponding L line (Fig. 3; results of ANOVAs: block 1, $F_{1,30}=5.17, P$ $<0.05$; block $2, F_{1,57}=21.25, P<0.001$; block $3, F_{1,51}=7.28$, $P<0.01$ ). Moreover, the grand mean ecdysteroid titer (mean of morph means in Fig. 3) was significantly elevated in the SW versus the LW(f) morph across days 3-5 of adulthood (Fig. 7; results of a paired $t$-test of least-squared grand mean titers averaged over the three days of adulthood: $\left.t_{2}=6.37, P<0.025\right)$. Grand mean titers of LW(f) and SW morphs (Fig. 7) also differed significantly on day $7\left(t_{2}=4.78, P<0.05\right)$, nearly differed significantly on day $5\left(t_{2}=3.21,0.1>P>0.05\right)$, and did not differ on day $3\left(t_{2}\right.$ $=1.51, P>0.2)$. These data strongly indicate that $\mathrm{LW}(\mathrm{f})$ females from the L lines differ genetically from SW females from the $\mathrm{S}$ lines with respect to the ecdysteroid titer.

\section{Discussion \\ Life-History Endocrinology: State of the Art}

The hormonal basis of life-history variation and trade-offs is a key issue in life-history physiology (Stearns 1989; Ketterson and Nolan 1992, 1999; Sinervo and Svensson 1998; Zera et al. 1998; Cisper and Zera 2001; Zera and Harshman 2001). Yet, there currently is only very limited information on any aspect of this topic. Especially relevant to this topic are direct measures of: (1) phenotypic and genetic variation for hormonal traits, such as hormone titers, receptor characteristics, or activities of hormone metabolizing enzymes; and (2) phenotypic and genetic correlations between endocrine and life-history traits. Although not sufficient (Zera and Harshman 2001), these two types of information are necessary for establishing a causal link between variation in a hormonal trait and variation in an individual life-history trait or a trade-off between traits. Indirect approaches to establishing a causal relationship between endocrine and life-history variation (e.g., using hormone manipulation) are fraught with problems unless they are used in conjunction with direct measures of in vivo hormonal variation (Zera et al. 1998; Zera and Cisper 2001; Zera and Harshman 2001).

Thus far, only a handful of studies have directly quantified naturally occurring phenotypic variation for hormone titers within species and associations between hormone titers and life-history traits or trade-offs (Moore 1986; Ketterson and Nolan 1992, 1999; Cisper et al. 2000; Sinervo et al. 2000). Most of these studies have focused on the endocrine basis of behavioral variation. Correlations between hormone titers and physiological or morphological components of life-history variation (e.g., onset of ovarian growth, trade-off between masses of reproductive vs. nonreproductive organs) are particularly lacking (Cisper et al. 2000).

Information on the endocrine-genetic basis of life-history variation and covariation is especially important for understanding how physiological regulation is modified during life-history evolution. Reports of naturally occurring genetic variation and covariation for hormonal regulators have mainly focused on the activity of the regulatory enzyme, juvenile hormone esterase, in species of crickets (e.g., Zera and Tiebel 1989; Zera and Zhang 1995; Roff et al. 1997; Zera and Huang 1999; Zera and Harshman 2001). Only rarely have studies quantified genetically based variation of in vivo hormone titers themselves (Kallman 1989; 

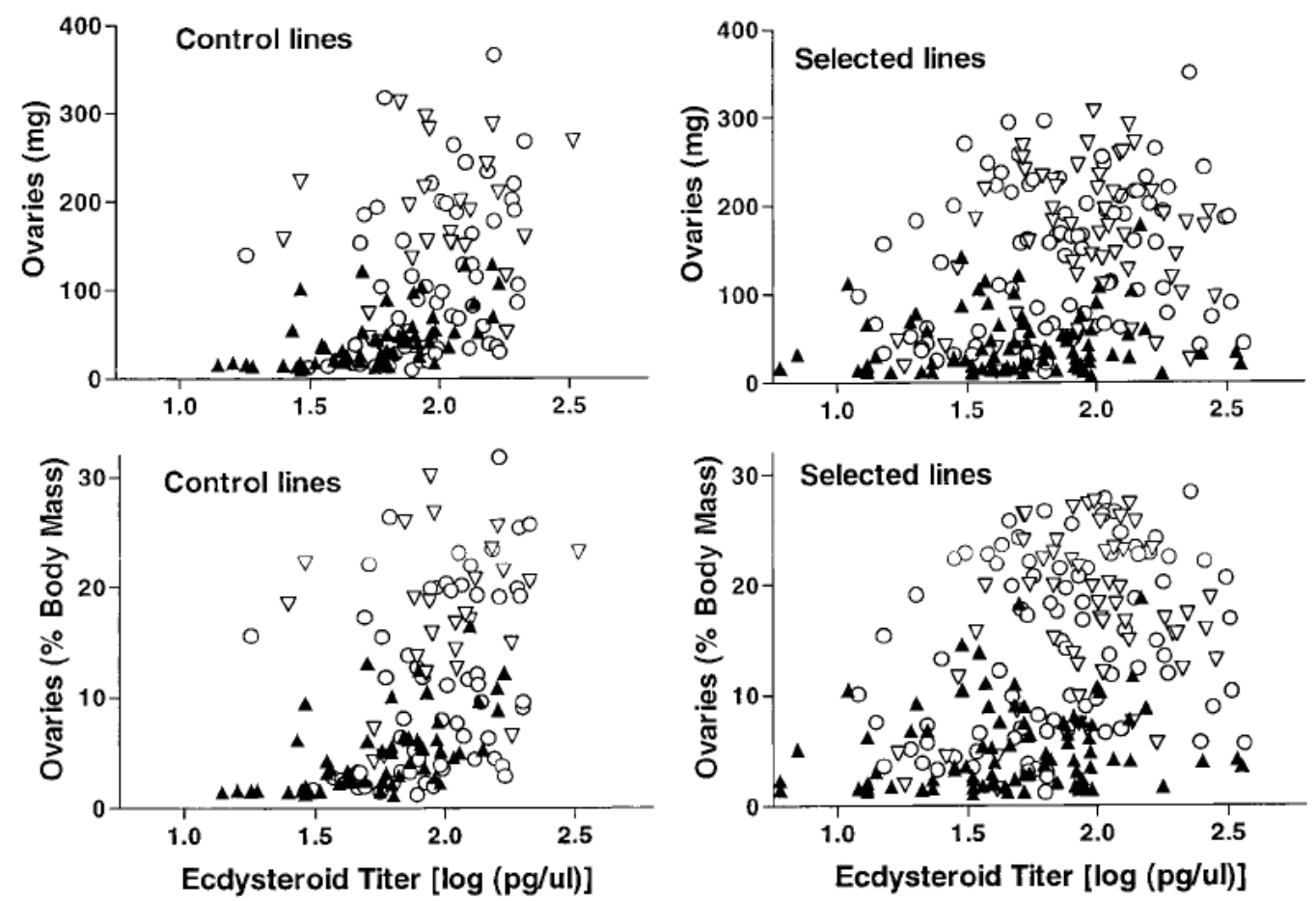

Figure 6. Bivariate distributions of ovarian mass (unscaled ovarian mass in top panels and ovarian mass scaled to total body mass in bottom panels) and $\log$ ecdysteroid titer in morphs. Morphs of Gryllus firmus were derived from control lines in left panels and from selected lines in right panels. Filled triangles, LW(f) (long-winged females with functional flight muscles); open triangles, LW(h) (long-winged females with histolyzed [reduced] flight muscles); open circles, SW (short-winged females, all with reduced flight muscles).

Zera and Cisper 2001; present study). Finally, no study, including the present one, has yet documented naturally occurring genetic variation for a hormonal receptor. Clearly, investigations of the endocrine underpinnings of life-history variation and tradeoffs are in their infancy.

\section{Ecdysteroid Titer Variation in Gryllus firmus: Existence and Potential Significance}

The present study (Table 1; Figs. 4, 5) and previous studies (Roff 1990a,b; Zera et al. 1997; Zera and Huang 1999) have estab- lished that G. firmus contains morphs that differ phenotypically and genetically in morphological aspects of flight capability (wing length, flight muscle development) and ovarian growth during the first week of adulthood. In the present study, we documented a consistent and parallel elevation in the ecdysteroid titer and ovarian mass in flightless [SW or LW(h)] versus flight-capable [LW(f)] morphs of the wing-polymorphic cricket G. firmus during the first week of adulthood. This was the case for phenotypic differences in the ecdysteroid titer and ovarian mass among morphs derived from the same control lines or from the same or different selected lines (Figs. 2-5). More importantly, we docu-

Table 1. Frequencies of wing and flight-muscle morphs in Gryllus firmus on various days of adulthood in selected and control lines. LW(f), flight-capable morph, with long wings and functional flight muscles; LW(h), flightless, long-winged morph with histolyzed flight muscles; SW, short-winged, flightless morph with underdeveloped flight muscles; L, LW-selected line; S, SW-selected lines; C, control (unselected lines). LW(f) and LW(h) morphs were not scored in S lines and therefore only the frequency of the composite long-winged (LW) morph is given. Morph frequencies were based on samples of 30-50 individuals scored during the sixth generation of selection. Day 0 was the day of adult molt.

\begin{tabular}{|c|c|c|c|c|c|c|c|c|}
\hline \multirow[b]{2}{*}{ Block } & \multirow[b]{2}{*}{ Morph } & \multicolumn{3}{|c|}{$\mathrm{L}$ lines } & \multirow{2}{*}{$\begin{array}{c}\text { S Lines } \\
\text { Days 3-7 }\end{array}$} & \multicolumn{3}{|c|}{$\mathrm{C}$ lines } \\
\hline & & Day 3 & Day 5 & Day 7 & & Day 3 & Day 5 & Day 7 \\
\hline 1 & $\begin{array}{l}\text { LW(f) } \\
\text { LW(h) } \\
\text { SW }\end{array}$ & $\begin{array}{l}0.75 \\
0.08 \\
0.17\end{array}$ & $\begin{array}{l}0.62 \\
0.15 \\
0.23\end{array}$ & $\begin{array}{l}0.62 \\
0.15 \\
0.23\end{array}$ & $\begin{array}{l}0.35(\mathrm{LW}) \\
0.65\end{array}$ & $\begin{array}{l}0.51 \\
0.07 \\
0.42\end{array}$ & $\begin{array}{l}0.49 \\
0.12 \\
0.39\end{array}$ & $\begin{array}{l}0.45 \\
0.19 \\
0.36\end{array}$ \\
\hline 2 & $\begin{array}{l}\text { LW(f) } \\
\text { LW(h) } \\
\text { SW }\end{array}$ & $\begin{array}{l}0.79 \\
0.00 \\
0.21\end{array}$ & $\begin{array}{l}0.67 \\
0.09 \\
0.24\end{array}$ & $\begin{array}{l}0.62 \\
0.18 \\
0.20\end{array}$ & $\begin{array}{l}0.30(\mathrm{LW}) \\
0.70\end{array}$ & & & \\
\hline 3 & $\begin{array}{l}\text { LW(f) } \\
\text { LW(h) } \\
\text { SW }\end{array}$ & $\begin{array}{l}0.83 \\
0.04 \\
0.13\end{array}$ & $\begin{array}{l}0.77 \\
0.12 \\
0.11\end{array}$ & $\begin{array}{l}0.72 \\
0.19 \\
0.09\end{array}$ & $0.37(\mathrm{LW})$ & $\begin{array}{l}0.45 \\
0.03 \\
0.52\end{array}$ & $\begin{array}{l}0.39 \\
0.08 \\
0.53\end{array}$ & $\begin{array}{l}0.36 \\
0.15 \\
0.49\end{array}$ \\
\hline
\end{tabular}



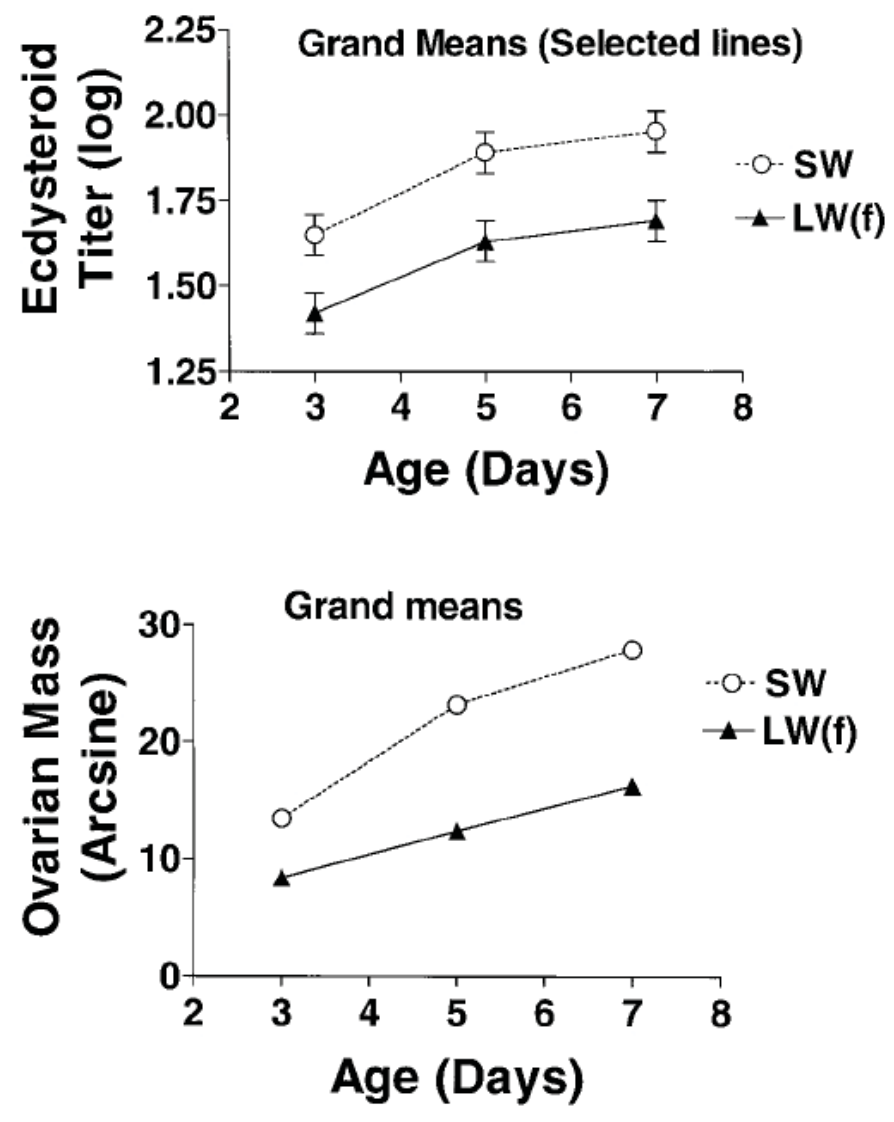

Figure 7. Genetic differences in ecdysteroid titers (upper panel) and ovarian mass (lower panel) between LW(f) and SW morphs of G. firmus during adulthood. Symbols represent grand means \pm SEM (see Methods for calculation). Symbols are the same as in Figure 1. Standard errors of ovarian masses were smaller than the symbols for the means. Ovarian masses were scaled to total body mass and very similar results were obtained on raw ovarian masses (data not shown; see Methods).

mented a genetically based elevation in these traits in the flightless SW versus the flight-capable LW(f) morph (Fig. 7, Results). Finally, highly significant, positive phenotypic correlations between the ecdysteroid titer and ovarian mass were observed in separate analyses of individual crickets derived from either control or selected lines (Results, Fig. 6). These robust results are consistent with the hypothesis that phenotypic and genetic differences in the ecdysteroid titer are important physiological causes of phenotypic and genetic differences in ovarian growth between morphs of G. firmus. This is one of the few studies directly documenting the existence of genetically based differences in the titer of a hormone between phenotypes that differ genetically in a lifehistory trait (also see Kallman 1989; Zera and Cisper 2001).

Our conclusions concerning the existence of genetically based differences in flight capability, ecdysteroid titer, and ovarian mass between LW(f) and SW morphs from selected lines are contingent upon the absence of strong maternal or paternal effects on these traits. All LW(f) individuals from L lines had LW parents, whereas all SW individuals from S lines had SW parents. However, for a variety of reasons, we consider maternal or paternal effects to be highly unlikely explanations for differences between morphs in the traits mentioned above. Genetic analyses of wing polymorphism in several species of Gryllus and related genera, which have included reciprocal crosses and backcrosses between LW-selected and SW-selected lines, have failed to find any significant maternal or paternal effect on wing morph expression (e.g., Modicogryllus confirmatus, Suzuki and Tanaka 1996; G. rubens, Zera and Rankin 1989; G. firmus, Roff 1990a; A. Zera, unpublished data). It is highly unlikely that traits such as the ecdysteroid titer and ovarian growth, which are highly correlated with wing morph, would be strongly affected by maternal or paternal influences while wing morph itself is not. Indeed, reciprocal crosses and backcrosses of selected lines provided no evidence for maternal or paternal effects on the activity of juvenile hormone esterase, an important endocrine regulator that is highly correlated with wing morph in Gryllus (Zera and Tiebel 1989; A. Zera, unpublished data).

Although ecdysteroids have not been shown to specifically regulate ovarian growth in G. firmus, it is highly likely that this is the case. Ecdysteroids are known to regulate many important aspects of reproduction in a wide variety of insects, including other crickets of the genus Gryllus (reviewed in Strambi et al. 1997). Important roles include stimulation of yolk protein synthesis and the regulation of development of previtellogenic follicles (Nijhout 1994; Strambi et al. 1997; Tawfik et al. 1997). The two- to threefold variation in mean (untransformed) ecdysteroid titers between adult morphs of G. firmus is similar to the magnitude of titer variation observed between castes or phases of functionally analogous polyphenisms in insects (Strambi et al. 1984; Zera et al. 1989; Rembold 1990; Tawfik et al. 1997). This degree of ecdysteroid titer variation is thought to be functionally important. Thus, differences in the ecdysteroid titer between morphs, as documented in the present study, have the potential to be functionally important with respect to morph-specific ovarian growth in G. firmus.

At present, there are two functional explanations for the difference in the ecdysteroid titer between the flight-capable and flightless morphs of G. firmus. First, the elevated ecdysteroid titer could directly cause of the faster egg development observed in the flightless morphs. Second, many important biochemical differences exist between the morphs (e.g., concentration of lipid flight fuels and yolk protein) that affect reproduction and that result from the synthesis of macromolecules in tissues other than the ovary (Zera et al. 1994, 1998). For example, yolk protein and lipids are synthesized in the fat body and are known to be regulated by a variety of hormones, including ecdysteroids (Kerkut and Gilbert 1985; Strambi et al. 1997; Zera et al. 1998). Thus, morph-specific ecdysteroid titers could coordinate the differing rates of yolk protein and lipid biosynthesis found in flight-capable and flightless morphs. Alternatively, ecdysteroids are synthesized in ovarian follicles (as well as in other tissues in Gryllus; Strambi et al. 1997) and are secreted into the hemolymph. Thus, it is also possible that the increased hemolymph ecdysteroid titer in flightless females could be a simple consequence of greater hormone secretion by their larger ovaries, without functional significance. Functional studies that investigate the effect of experimentally altered ecdysteroid titers on egg growth and other morph-specific traits are the next 
important step in establishing the causal role of ecdysteroids in life-history variation in G. firmus.

Morphs that differ in the ecdysteroid titer also differ in two dispersal characters, the length of the wings and the size of the flight muscles (Figs. 2, 3, 7). However, morph-specific differences in the adult ecdysteroid titer are unlikely to cause differences in either of these dispersal traits. Divergence in the development of wings and flight muscles between LW(f) and SW morphs begins during the juvenile stage (Pener 1985; Nijhout 1994), well before the differences in the adult ecdysteroid titer are manifest. Differences in flight muscle histolysis between LW(f) and $\mathrm{LW}(\mathrm{h})$ morphs arise during the same period in adulthood when these morphs differ in the ecdysteroid titer. However, experimental studies in several cricket species, including G. firmus, indicate that juvenile hormone, rather than ecdysteroids, likely regulates the production of the $\mathrm{LW}(\mathrm{h})$ morph via flight-muscle histolysis (Chudakova et al. 1982; Tanaka 1994; Zera and Cisper 2001).

\section{Additional Evolutionary Implications of the Present Study}

\section{Physiological similarities between the two flightless morphs and the evolution of flightlessness}

Gryllus firmus exhibits the two common types of flightlessness found in insects (Zera and Denno 1997). The SW morph has both reduced wings and flight muscles, produced by blockage of the growth of these organs in juveniles, whereas the LW(h) morph has fully developed wings, but reduced flight muscles, produced by muscle histolysis during adulthood. To what extent do these flightless morphs differ from each other and the flight-capable morph in physiological and reproductive traits? The extent of this differentiation will have a strong effect on which mode of flightlessness is favored by natural selection (Zera et al. 1997).

Results of the present study indicate no strong difference between the SW and LW(h) morphs in either the ecdysteroid titer or ovarian growth. Ovarian mass was very similar for the two flightless morphs on all days in both control and selected lines and differed dramatically from the LW(f) morph (Figs. 4, 5). A similar situation occurred for the hemolymph ecdysteroid titer (Figs. 2, 3). The only exceptions were titers on days 5 and 7 in the C-1 line, which were lower in LW(h) than in SW females and were very similar to the LW(f) morph (Fig. 2). However, these differences were due primarily to a few unusually low titer values in LW(h) females of the C-1 line on those days. The similar divergence in reproductive and physiological characteristics for either the LW(h) or SW morph compared with the LW(f) morph is consistent with results of earlier physiological comparisons among these morphs. For example, both flightless morphs exhibited very similar enzyme activities and respiration rates of flight muscles (Zera et al. 1997, 1999), various indices of nutrient assimilation and conversion (Zera and Brink 2000), and hemolymph juvenile hormone titers (Zera and Cisper 2001). All of these traits differed significantly between either flightless morph and the flight-capable morph. These data collectively indicate that any advantage in the evolution of flightlessness by blockage of flight-muscle growth in juveniles versus histolysis of grown muscles in adults must result from differences between the two flightless morphs in characteristics other than the ones listed above. Possibilities that have yet to be investigated include increased energy saving by the SW morph during the juvenile stage due to absence of flight muscle growth. This could be an important advantage over the LW(h) morph in nutrient-poor environments. Alternatively, the ability of LW(h) adults to disperse by flight prior to muscle histolysis could be an important advantage over SW adults in unpredictable environments.

\section{Effect of genetic background on morph characteristics}

An important issue in evolutionary studies of morphological polymorphism, such as wing polymorphism, is the extent to which morph characteristics are invariant features of the morph, rather than being dependent upon genetic background. For example, Roff (1994) reported that the fecundities of morphs of G. firmus exhibited negative frequency dependence; that is, the fecundity of a particular morph was negatively correlated with its frequency in selected lines. This result has interesting evolutionary implications, because it implies that the fixation of a morph will be opposed by its decreasing fecundity as its frequency increases.

In the present study, we tested for effects of genetic background on the ecdysteroid titer by comparing the titer in each morph derived from selected versus control lines that differed in morph frequencies (see Methods). We observed no or only a very small effect of genetic background on the ecdysteroid titer in the LW(f) or SW morphs and a significant effect in the LW(h) morph in only one of the two blocks (Results). As mentioned above, this significant difference for the LW(h) morph may have been influenced by a few unusual titer values. These data suggest that hormone titers may be inherent features of the morphs rather than depending upon the genetic background. However, our tests were not as statistically powerful as those in Roff (1994), because the lines that he used differed to a greater degree in morph frequencies than did ours. The effect of genetic background on morphspecific endocrine features is an important topic that deserves attention in future studies.

\section{Similarities between phenotypic and genetic variation in the ecdysteroid titer}

The extent to which phenotypic variation or covariation can be used as surrogates for genetic variation or covariation in studies of life-history evolution is currently a subject of debate (Reznick 1985, 1992; Partridge 1992; Leroi et al. 1994; Rose et al. 1996). Phenotypic studies are much simpler to conduct than genetic studies, which require large sample sizes in sibling breeding, parent-offspring regression, or artificial selection experiments. However, phenotypic and genetic variance for the same trait can differ markedly and genetic and phenotypic correlations measured on the same pairs of traits can differ in sign (Falconer and Mackay 1996). In such cases, misleading inferences concerning the evolution of traits might be drawn from studies of their phenotypic variation or covariation. The extent to which phenotypic variance and covariance approximate genetic variance and covariance is an empirical issue that can only be resolved by direct comparison, as has been done for comparisons between phenotypic and genetic correlations for morphological traits (Roff 1997). Data on genetic and phenotypic variation for hormone titers have become available only very recently. Phenotypic differences among the morphs of G. firmus in the ecdysteroid titer 
were very similar to genetic differences (Figs. 2-4). It is currently unknown whether the strong correspondence is due to similar influences of environmental and genetic effects on the phenotypic variation or to high heritabilities for the hormone titers, in which case the phenotypic variance would be primarily composed of genetic variance. Whatever the reason, results of the present study at the very least indicate that phenotypic differences in the ecdysteroid titer between morphs of G. firmus can be used as a surrogate for genetic differences the titer of this hormone between morphs. There currently are no estimates of genetic correlations for hormone titers in natural populations of any organism, thus precluding an assessment of the degree of similarity between genetic and phenotypic correlations involving the same hormone titer.

In summary, we have documented that naturally occurring flight-capable and flightless morphs of the cricket G. firmus exhibit phenotypic and genetic differences in an important life-history trait, early ovarian growth, that parallel phenotypic and genetic differences in the titer of a key reproductive hormone that potentially regulates these reproductive differences. This represents and important step in identifying the endocrine causes of life-history variation.

\section{Acknowledgments}

Research reported here was supported by grant 9507388 from the National Science Foundation. We thank E. Chang (Bodega Marine Laboratory, Bodega Bay, California) for supplying us with the ecdysteroid antiserum.

\section{Literature Cited}

Calow P. 1979. The cost of reproduction: a physiological approach. Biol. Rev. 54:23-40.

Chang E. S., J. D. O'Connor. 1979. Arthropod molting hormones. Pp. 797-814 in B. M. Jaffe and H. R. Behrman, eds. Methods of hormone radioimmunoassay. Academic Press, New York.

Chudakova I., V. Maslennikova, E. Luchnikova. 1982. Effect of 20hydroxyecdysone on Acheta domesticus L. (Orthoptera) and Drosophila melanogaster Meig (Diptera) reproduction. Zool. Jb. Physiol. 86:45-52.

Cisper G., A. J. Zera, D. W. Borst. 2000. Juvenile hormone titer and morph-specific reproduction in the wing-polymorphic cricket, Gryllus firmus. J. Insect Physiol. 46:585-596.

Djawdan M., T. T. Sugiyama, L. K. Schlaeger, T. J. Bradley, M. R. Rose. 1996. Metabolic aspects of the trade-off between fecundity and longevity in Drosophila melanogaster. Physiol. Zool. 69:1176-1195.

Falconer D. S., T. F. C. Mackay. 1996. Introduction to quantitative genetics. 4th ed. Longman Scientific and Technical, New York.

Fisher R. A. 1930. The genetical theory of natural selection. Dover, New York.

Harrison R. G. 1980. Dispersal polymorphisms in insects. Annu. Rev. Ecol. Syst. 11:95-118.

Horn D. H. S., J. S. Wilkie, B. A. Sage, J. D. O’Connor. 1976. A high-affinity antiserum specific for the ecdysone nucleus. J. Insect Physiol. 22:901-905.

Kallman K. 1989. Genetic control of size at maturity in Xiphophorus.. Pp. 163-184 in G. K. Meffe and F. F. Snelson, Jr., eds. Ecol- ogy and evolution of live-bearing fishes (Poeciliidae). Prentice Hall, Englewood Cliffs, NJ.

Kerkut G. A., L. I. Gilbert. eds. 1985. Comprehensive insect biochemistry, physiology and pharmacology. Vols. 7, 8. Endocrinology. Pergamon Press, Oxford, U.K.

Ketterson E. D., V. Nolan Jr. 1992. Hormones and life histories: an integrative approach. Am. Nat. 140:33-62 S.

Ketterson E. D. 1999. Adaptation, exaptation and constraint. Am. Nat. 154:4-25 S.

Leroi A. M., B. K. Sung, M. R Rose. 1994. The evolution of phenotypic life history trade-offs: an experimental study using Drosophila melanogaster. Am. Nat. 144:661-676.

Martin C. R. 1985. Endocrine physiology. Oxford Univ. Press, New York.

Moore M. C. 1986. Elevated testosterone levels during nonbreedingseason territoriality in a fall-breeding lizard, Sceloporus jarrovi. Comp. Physiol. A. 158:159-163.

Nijhout H. F. 1994. Insect hormones. Princeton Univ. Press, Princeton, NJ.

Norris D. 1996. Vertebrate endocrinology. Lea and Fabigin, Philadelphia, PA.

Packard G., T. J. Bordman. 1987. The misuse of ratios to scale physiological data that vary allometrically with body size. Pp. 216236 in M. E. Feder, A. F. Bennett, W. W. Burggren, and R. B. Huey, eds. New directions in ecological physiology. Cambridge Univ. Press, Cambridge, U.K.

Partridge L. 1992. Measuring reproductive costs. Trends Ecol. Evol. 7:99-100.

Pener M. P. 1985. Hormonal effects on flight and migration. Pp 491550 in G. A. Kerkut and L. I. Gilbert, eds. Comprehensive insect biochemistry, physiology, and pharmacology. Vol. 7. Pergamon, Oxford, U.K.

Rembold H. 1990. Roles of morphogenetic hormones in caste polymorphism is sting bees. Pp. 325-345 in A. P. Gupta, ed. Morphogenetic hormones of arthropods. Vol. 3. Roles in histogenesis, organogenesis, and morphogenesis. Rutgers Univ. Press, New Brunswick, NJ.

Reznick D. N. 1985. Cost of reproduction: an evaluation of experimental evidence. Oikos. 44:257-267.

Reznick D. N. 1992. Measuring the costs of reproduction. Trends Ecol. Evol. 7:42-45.

Roff D. A. 1986. The evolution of wing dimorphism in insects. Evolution. 40:1009-1020.

Roff D. A. 1990a. Selection for changes in the incidence of wing dimorphism in Gryllus firmus. Heredity. 65:163-168.

Roff D. A. 1990b. Antagonistic pleiotropy and the evolution of wing dimorphism in the sand cricket, Gryllus firmus. Heredity. 65:169-177.

Roff D. A. 1994. Evidence that the magnitude of the trade-off in a dichotomous trait is frequency dependent. Evolution. 48:1650 1656.

Roff D. A. 1997. Evolutionary quantitative genetics. Chapman and Hall, New York.

Roff D. A., G. Stirling, D. J. Fairbairn. 1997. The evolution of threshold traits: a quantitative genetic analysis of the physiological and life-history correlates of wing dimorphism in the sand cricket. Evolution. 51:1910-1919. 
Rose M. R., T. J. Nusbaum, A. K. Chippendale. 1996. Laboratory evolution: the experimental wonderland and the Cheshire Cat syndrome. Pp 221-244 in M. R. Rose and G. V. Lauder, eds. Adaptation. Academic Press, San Diego, CA.

Sinervo B., E. Svensson. 1998. Mechanistic and selective causes of life history trade-offs and plasticity. Oikos. 83:432-442.

Sinervo B., D. B. Miles, W. A. Frankino, M. Klukowski, D. F. DeNardo. 2000. Testosterone, endurance, and Darwinian fitness: natural and sexual selection on the physiological bases of alternate male behaviors in side-blotched lizards. Horm. Behav.: 38:222-233.

Sokal R. R., F. J. Rohlf. 1989. Biometry. 2d ed. W. H. Freeman and Company, San Francisco, CA.

Stearns S. C. 1989. Trade-offs in life-history evolution. Funct. Ecol. 3:259-268.

Stearns S. C. 1992. The evolution of life histories. Oxford Univ. Press, Oxford, U.K.

Strambi A., C. Strambi, P.-F. Rosler, I. Rosler. 1984. Simultaneous determination of juvenile hormone and ecdysteroid titers in the hemolymph of bumblebee prepupae (Bombus hypnorum and $B$. terrestris). Comp. Gen. Endocrinology. 55:83-88.

Strambi A., C. Strambi, M. Cayre. 1997. Hormonal control of reproduction and reproductive behavior in crickets. Arch. Insect Biochem. Physiol. 35:393-404.

Suzuki Y., S. Tanaka. 1996. Environmental and genetic control of wing morph determination in three subtropical populations of a cricket, Modicogryllus confirmatus (Orthoptera: Gryllidae). Jpn. J. Ent. 64:896-909.

Tanaka S. 1994. Endocrine control of ovarian development and flight muscle histolysis in a wing dimorphic cricket, Modicogryllus confirmatus. J. Insect Physiol. 40:483-490.

Tawfik A. I., A. Vedrova, W. Li, F. Sehnal, D. Obeng-Ofori. 1997. Haemolymph ecdysteroids and the prothoracic glands in the solitary and gregarious adults of Schistocerca gregaria. J. Insect Physiol. 43:485-493.

Towsend C. R., P. Calow. 1981. Physiological ecology: an evolutionary approach to resource use. Blackwell Scientific Publications, Oxford, U.K.

Veazy J. N., C. A. R. Kay, T. J. Walker, W. H. Whitcomb. 1976. Seasonal abundance, sex ratio, and macroptery of field crickets in northern Florida. Ann. Entomol. Soc. Am. 69:374-380.

Warren J. T., W. Smith, L. I. Gilbert. 1984. Simplification of the ecdysteroid radioimmunoassay by the use of protein A from Staphylococcus aureus. Experientia. 40:393-394.

Wyatt G. R., K. G. Davey. 1996. Cellular and molecular actions of juvenile hormone. II. Roles of juvenile hormone in adult insects. Adv. Insect Physiol. 26:1-155.
Zera A. J., T. Brink. 2000. Nutrient absorption and utilization by wing and flight muscle morphs of the cricket Gryllus firmus: implications for the trade-off between flight capability and early reproduction. J. Insect Physiology. 46:1207-1218.

Zera A. J., G. Cisper. 2001. Genetic and diurnal variation in the juvenile hormone titer in a wing-polymorphic cricket: implications for the evolution of life histories and dispersal. Physiol. Biochem. Zool. In Press. .

Zera A. J., R. F. Denno. 1997. Physiological and ecological aspects of dispersal polymorphism in insects. Annu. Rev. Entomol. 42:207231.

Zera A. J., L. G. Harshman. 2001. The physiology of life history trade-offs in animals. Annu. Rev. Ecol. Syst. 31 in Press.

Zera A. J., Y. Huang. 1999. Evolutionary endocrinology of juvenile hormone esterase: functional relationship with wing polymorphism in the cricket, Gryllus firmus. Evolution: 53:837-847.

Zera A. J., M. A. Rankin. 1989. Wing dimorphism in Gryllus rubens: genetic basis of morph determination and fertility differences between morphs. Oecologia. 80:249-1255.

Zera A. J., K. C. Tiebel. 1989. Differences in juvenile hormone esterase activity between presumptive macropterous and brachypterous Gryllus rubens: implications for the hormonal control of wing polymorphism. J. Insect Physiol. 35:7-17.

Zera A. J., C. Zhang. 1995. Direct and correlated responses to selection on hemolymph juvenile hormone esterase activity in Gryllus assimilis.. Genetics. 141:1125-1134.

Zera A. J., C. Strambi, K. C. Tiebel, A. Strambi, M. A. Rankin. 1989. Juvenile hormone and ecdysteroid titers during critical periods of wing morph determination in Gryllus rubens.. J. Insect Physiol. 35:501-511.

Zera A. J., S. Mole, K. Rokke. 1994. Lipid, carbohydrate and nitrogen content of long- and short-winged Gryllus firmus: implications for the physiological cost of flight capability. J. Insect Physiol. 40:1037-1044.

Zera A. J., J. Sall, K. Grudzinski. 1997. Flight-muscle polymorphism in the cricket Gryllus firmus: muscle characteristics and their influence on the evolution of flightlessness. Physiol. Zool. 70:519529.

Zera A. J., J. Potts, K. Kobus. 1998. The physiology of life history trade-offs: experimental analysis of a hormonally-induced life history trade-off in Gryllus assimilis.. Am. Nat. 152:7-23.

Zera A. J., J. Sall, K. Otto. 1999. Biochemical aspects of flight and flightlessness in Gryllus: flight fuels, enzyme activities and electrophoretic profiles of flight muscles from flight-capable and flightless morphs. J. Insect Physiol. 45:275-285. 